\title{
Massive pulmonary arteriovenous fistula in the newborn
}

\author{
C. P. Clarke, T. H. Goh, A. Blackwood, and A. W. Venables \\ From the Division of Cardiac Surgery and Department of Cardiology, Royal Children's Hcspital, Melbourrie, \\ Australia
}

Although pulmonary arteriovenous fistula as a cause of cyanosis is well recognized, most of the reported cases occur in older children and adults, and its importance as a correctable lesion in the newborn is often overlooked. The details of two babies who presented with cyanosis in the first few days of life are presented to emphasize that this eminently treatable lesion may need to be managed as an emergency.

Since the first description of pulmonary arteriovenous fistula (Churton, 1897), several large series have been collected (Bosher, Blake, and Byrd, 1959; Moyer, Glantz, and Brest, 1962; Dines et al., 1974), and the importance of surgery to forestall paradoxical embolism, cerebral abscess, or rupture of the abnormal lung vessels, has been emphasized. Cyanosis commonly accompanies this lesion, but is not evident usually until later childhood, and most operations have been performed in adults.

The only report of a pulmonary arteriovenous fistula successfully treated in the neonatal period is that by Hall et al. in 1965. Reported here are 2 further cases of neonates presenting in the first few days of life with cyanosis caused by pulmonary arteriovenous fistula, showing that this lesion may need to be treated as a surgical emergency.

\section{Case reports}

\section{Case 1}

A normal term male infant was noticed to be centrally cyanosed some hours after delivery, and there was an easily heard systolic murmur in the right chest, loudest at the base posteriorly.

A plain chest $x$-ray film showed that the heart was enlarged, there was a rounded mass behind the lower part of the right heart shadow, and the lung fields were relatively oligaemic (Fig. 1). An electrocardiogram showed a normal axis with left ventricular dominance. The haemoglobin was $18 \cdot 1 \mathrm{~g} / \mathrm{dl}$.

Cardiac catheterization showed normal pressures

Received 25 February 1976. except for a low diastolic pressure in the pulmonary artery. Oxygen saturation of a blood sample from the left atrium was 89 per cent, from the right upper pulmonary vein 97 per cent, and from the right lower pulmonary vein 42 per cent. A pulmonary angiogram (Fig. 2) showed a dilated right pulmonary artery which filled a sac-like structure communicating directly with the left atrium.

Shortly after this investigation the baby's condition deteriorated considerably, with increasing acidosis, and an emergency operation was undertaken through a right posterolateral thoracotomy. The main pulmonary artery was encircled and controlled with a tourniquet while an attempt was made to dissect the mass, but it was found that in order to dissect it properly, the right lower lobe had to be removed. The sac was then opened and the pulmonary artery closed from within. The single vein draining from the sac into the left atrium was ligated.

He did not improve after operation and died some hours later despite all attempts at resuscitation. A postmortem study of the heart showed that there was also a patent foramen ovale and a moderate sized persistent ductus arteriosus.

\section{Case 2}

A male baby weighing $3500 \mathrm{~g}$ at birth was delivered normally. Initially his condition appeared satisfactory, but 3 days later he was noted to have blue lips, and then became progressively more cyanosed though he continued to feed well and was not in any respiratory distress. On examination he 

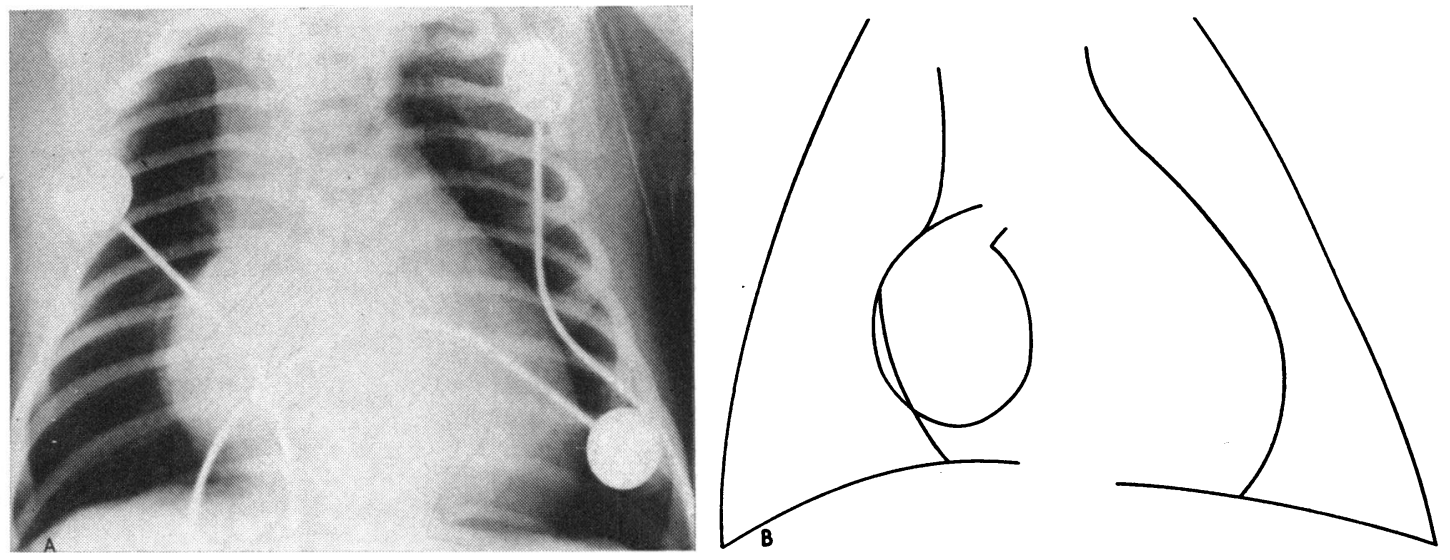

FIG. 1 Plain chest $x$-ray film of Case 1 ( $A$ ) showing a mass abutting the right heart shadow.

This is outlined in the accompanying line drawing $(B)$.

was centrally cyanosed even in oxygen, and a soft systolic murmur was heard in the left infraclavicular area. A chest $x$-ray film (Fig. 3) showed a normal sized heart with oligaemic lung fields and a ribbonlike shadow in the left mid zone descending to a rounded mass in the posterior region of the left lower lobe. The electrocardiogram showed RS complexes in V1 and V4R and a dominant $R$ wave in V6, a pattern suggestive of hypoplasia of the right ventricle. The haemoglobin was $19 \mathrm{~g} / \mathrm{dl}$, and the packed cell volume 61 per cent.

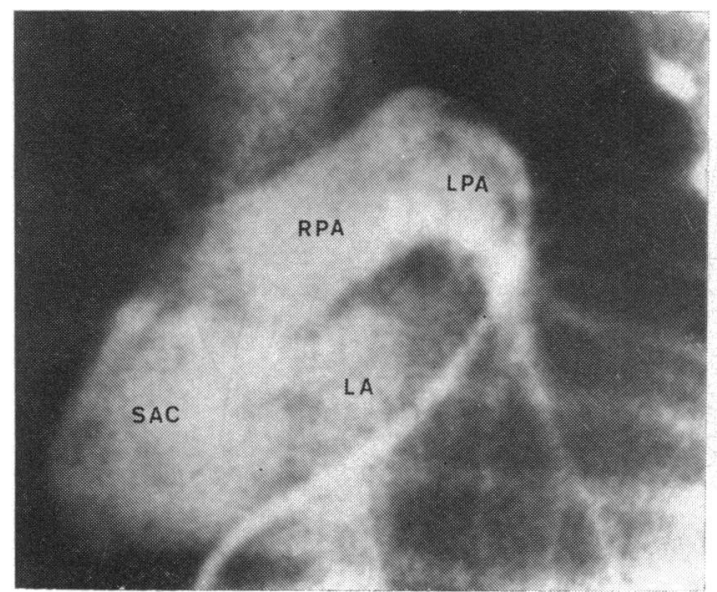

FI G. 2 Pulmonary angiogram of Case 1 showing rapid passage of dye from the right pulmonary artery (RPA) to a sac-like structure ( $S A C$ ) and then into the left atrium (LA). The left pulmonary artery (LPA) was of normal size.
Cardiac catheterization showed normal pressures and reduced oxygen saturation of samples from the left atrium. A pulmonary angiogram (Fig. 4) showed a large dilated vessel coming off the left pulmonary artery which passed down to a saccular structure in the lower part of the left lower lobe from which dye rapidly ascended through a dilated pulmonary vein to the left atrium. The catheter also passed across a patent foramen ovale, and there was a moderate sized persistent ductus arteriosus. An echocardiogram was normal.

After our previous experience (Case 1), and because cyanosis was increasing, an operation was performed on the 4th day of life through a left posterolateral thoracotomy. A moderate sized persistent ductus was ligated and a pulsatile mass in the posterior part of the left lower lobe was seen. This was fed by a large discrete branch of the left pulmonary artery with a single large vein draining from it directly to the left atrium. These structures were ligated, but the mass remained pulsatile and was, therefore, removed by resecting the posterior basal segment of the left lung.

The postoperative course was uneventful, and he was discharged from hospital 11 days later. Pathological examination of the resected lung segment showed a large number of vessels draining into a saccular structure which was considered to be an arteriovenous malformation.

\section{Discussion}

The majority of reported patients with pulmonary arteriovenous fistulae are adults (Moyer et al., 1962), but the symptoms may develop in early 

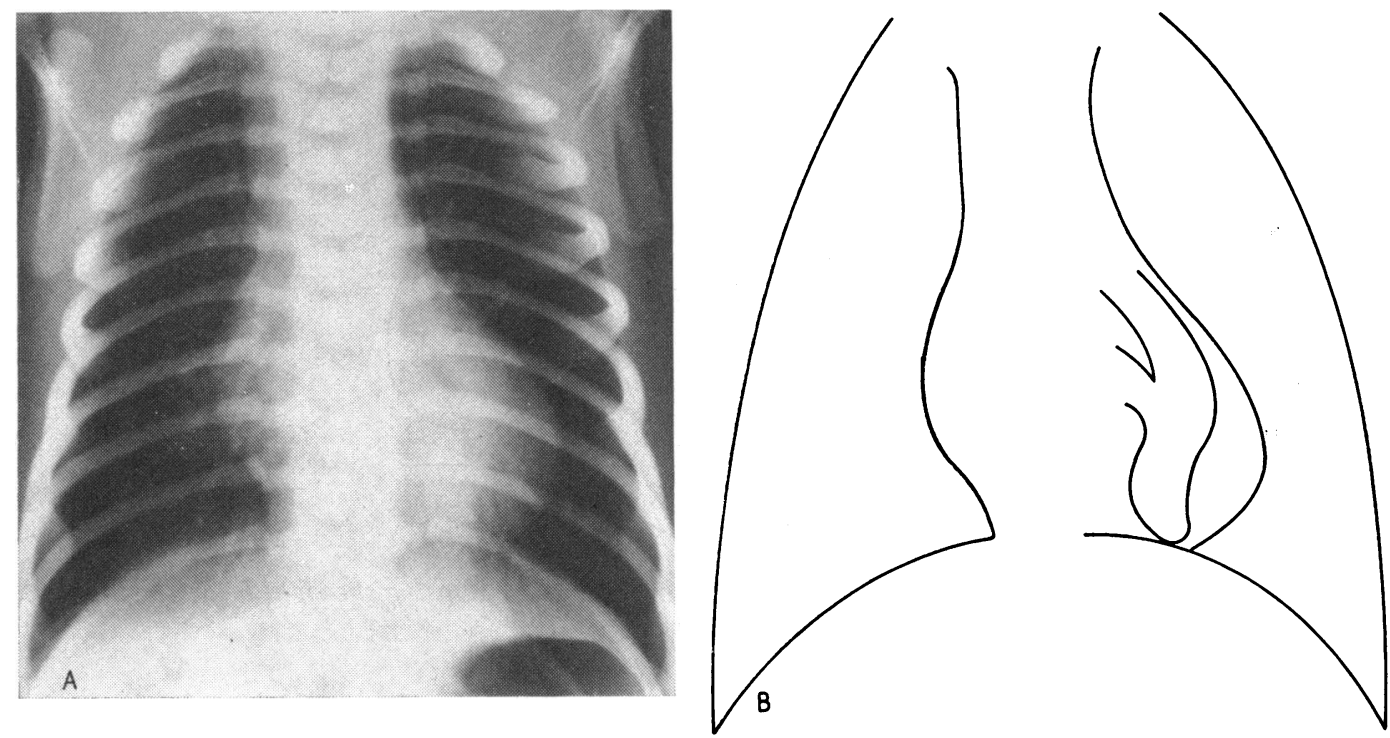

FIG. 3 Plain chest $x$-ray film of Case 2 ( $A$ ) showing a mass in the lower left lobe behind the heart shadow. This is detailed in the accompanying line drawing $(B)$.
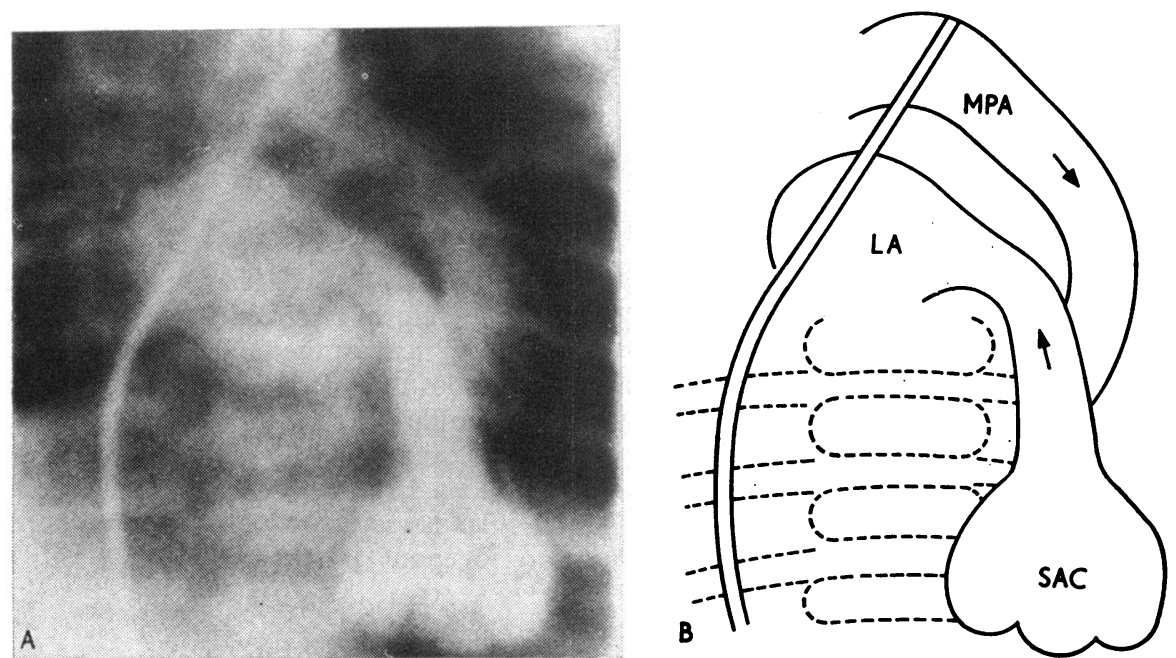

FIG. 4 (A) Pulmonary angiogram of Case 2 showing a rounded mass in the left lower lobe filling from the pulmonary artery and draining from a separate vein to the left atrium. This is illustrated in the accompanying line drawing $(B)$. 
life and in many cases appear to have been overlooked in infancy and childhood (Jeresaty, Knight, and Hart, 1966). Characteristically these patients are noted to be mildly cyanosed in childhood, and become increasingly cyanosed and clubbed as they grow older. While the physical examination may suggest a cardiac lesion, the malformation is often easily seen on a plain chest $x$-ray film, and can be precisely diagnosed by angiographic methods. Between a third and a half of all cases are associated with hereditary telangiectasia which is more common amongst those with multiple fistulae than single. The few cases treated in childhood have been well reviewed by Shumaker and Waldhausen (1963) and by Jeresaty et al. (1966), who advocate early excision of the lesion even if asymptomatic, to forestall complications such as emboli, brain abscess, and haemorrhage. The case reported by Hall et al. (1965) and the two reported here underline the point that this anomaly may need to be treated as an emergency when it presents in the neonatal period. A patient similar to our first case has been reported by Lucas, Lund, and Edwards (1961). He presented with cyanosis when aged 1, and died of a cerebral abscess 2 years later. Necropsy showed an almost direct communication between the right pulmonary artery and the left atrium via an aneurysmal sac.

Pulmonary arteriovenous fistulae do not usually cause an increase in cardiac output, and the right heart pressures are normal (Moyer et al., 1962). Cyanosis results from a significant shunt of blood through the low pressure fistula in preference to the pulmonary parenchyma, and implies a major lesion. As Hall et al. (1965) pointed out, when the low resistance pulmonary bypass has existed throughout intrauterine life counteracting the effects of the normal high fetal pulmonary vascular resistance, an unusual volume load is imposed on the left ventricle. This is increased if after birth the ductus arteriosus remains patent, as was the case in our two patients.

Although the combination of central cyanosis, oligaemic lung fields, and electrocardiographic evidence of left ventricular preponderance suggests a diagnosis of tricuspid atresia or hypoplastic right ventricle, an echocardiogram will rule out these possible diagnoses by showing a normal right ventricle. Careful review of the plain chest $x$-ray film may show the intrapulmonary opacity, and allow the true diagnosis to be made.

\section{References}

Bosher, L. H., Jr., Blake, D. A., and Byrd, B. R. (1959). An analysis of the pathologic anatomy of pulmonary arteriovenous aneurysms with particular reference to the applicability of local excision. Surgery, 45, 91.

Churton, T. (1897). Multiple aneurysm of pulmonary artery. British Medical fournal, 1, 1223.

Dines, D. E., Arms, R. A., Bernatz, P. E., and Gomes, M. R. (1974). Pulmonary arteriovenous fistulas. Proceedings of the Mayo Clinic, 49, 460.

Hall, R. J., Nelson, W. P., Blake, H. A., and Geiger, J. P. (1965). Massive pulmonary arteriovenous fistula in the newborn. A correctable form of 'cyanotic heart disease'. An additional cause of cyanosis with left axis deviation. Circulation, 31, 762.

Jeresaty, R. M., Knight, H. F., and Hart, W. E. (1966). Pulmonary arteriovenous fistulas in children. Report of two cases, and review of literature. American fournal of Diseases of Children, 111, 256.

Lucas, R. V., Jr., Lund, G. W., and Edwards, J. E. (1961). Direct communication of a pulmonary artery with the left atrium. An unusual variant of the pulmonary arteriovenous fistula. Circulation, 24, 1409.

Moyer, J. H., Glantz, G., and Brest, A. N. (1962). Pulmonary arteriovenous fistulas. Physiologic and clinical considerations. American fournal of Medicine, 32, 417.

Shumaker, H. B., Jr., and Waldhausen, J. A. (1963). Pulmonary arteriovenous fistulas in children. Annals of Surgery, 158, 713.

Requests for reprints to Mr. C. P. Clarke, Division of Cardiac Surgery, Royal Children's Hospital, Parkville, Victoria 3052, Australia. 